NASA Technical Memorandum 105808

AIAA-93-4400

\title{
Cruise Noise Measurements of a Scale Model Advanced Ducted Propulsor
}

James H. Dittmar and Christopher E. Hughes

Lewis Research Center

Cleveland, Ohio

Lawrence A. Bock

Pratt \& Whitney

United Technologies Incorporated

East Hartford, Connecticut

and

David G. Hall

Sverdrup Technology, Inc.

Lewis Research Center Group

Brook Park, Ohio

Prepared for the

15th AIAA Aeroacoustics Conference

sponsored by the American Institute of Aeronautics and Astronautics

Long Beach, California, October 25-27, 1993

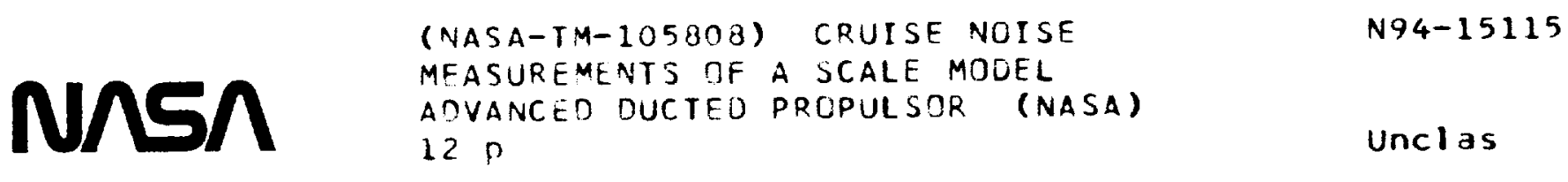





\title{
CRUISE NOISE MEASUREMENTS OF A SCALE MODEL ADVANCED DUCTED PROPULSOR
}

\author{
James H. Dittmar and Christopher E. Hughes \\ National Aeronautics and Space Administration \\ Lewis Research Center \\ Cleveland, Ohio \\ Lawrence A. Bock \\ Pratt \& Whitney \\ United Technologies Incorporated \\ East Hartford, Connecticut \\ and \\ David G. Hall \\ Sverdrup Technology, Inc. \\ Lewis Research Center Group \\ Brook Park, Ohio
}

\section{Abstract}

A scale model Advanced Ducted Propulsor (ADP) was tested in NASA Lewis Research Center's 8- by 6-Foot Wind Tunnel to obtain acoustic data at cruise conditions. The model, designed and manufactured by Pratt \& Whitney Division of United Technologies, was tested with three inlet lengths. The model has 16 rotor blades and 22 stator vanes, which results in a cut-on condition with respect to rotor-stator interaction noise. Comparisons of the noise directivity of the ADP with that of a previously tested high-speed, unducted propeller showed that the ADP peak blade passing tone was about $30 \mathrm{~dB}$ below that of the propeller, and therefore, should not present a cabin or enroute noise problem. The maximum blade passing tone first increased with increasing helical tip Mach number, peaked, and then decreased at a higher Mach number. The ADP tests with the shortest inlet showed more noise in the inlet arc than did tests with either of the other two inlet lengths.

\section{Introduction}

The National Aeronautics and Space Administration recently completed a program demonstrating that significant improvements in fuel efficiency are achievable by powering aircraft with advanced turboprops instead of with equivalent technology, turbofans. ${ }^{1}$ The cruise noise caused by the advanced, high-speed propellers on these aircraft could present a cabin environment or ground (enroute) noise problem. High noise levels on the fuselage could require excess acoustic treatment in the airplane wall to provide acceptable interior sound levels. High enroute levels could impact aircraft design or operation. The cruise noise of these advanced turboprop models was previously measured in NASA Lewis Research Center's 8- by 6-Foot Wind Tunnel ${ }^{2}$ and estiCopyright 1993 by the American Institute of Aeronautics and Astronautics, Inc. No copyright is asserted in the

United States under Title 17, U.S. Code. The U.S. Govern-

ment has a royalty-free license to exercise all rights under

the copyright claimed herein for Governmental purposes.

All other rights are reserved by the copyright owner. mates of the enroute noise levels were made. ${ }^{3}$ The fuselage noise reduction benefits of installing an advanced propeller in a short duct were also estimated.

For aircraft installation reasons, particularly in large thrust sizes, the current emphasis in the NASA subsonic aeropropulsion program is on very high bypass ratio turbofans. A candidate configuration is a ducted propeller that combines turbofan and propeller technologies. This device features a variable pitch rotor, with downstream stator vanes, contained in a nacelle. As part of this effort, a scale model Advanced Ducted Propulsor (ADP), designed and built by Pratt \& Whitney Division of United Technologies, was tested at cruise conditions in the NASA Lewis 8- by 6-Foot Wind Tunnel (Fig. 1). Acoustic data were previously obtained on this model at the takeoff and landing conditions. ${ }^{5}$ In Ref. 5 , the beneficial acoustic effects of cutoff on rotor-stator interaction noise were demonstrated. The cruise noise levels obtained in the 8- by 6-Foot Wind Tunnel are presented in this paper for the ADP model with three inlet duct lengths.

\section{Apparatus and Procedure}

\section{Advanced Ducted Propulsor}

The Advanced Ducted Propulsor (ADP) model has 16 rotor blades and is $43.8 \mathrm{~cm}$ (17.25 in.) in diameter (Fig. 1). Table I presents some design parameters for this model. The ADP design has two interchangeable sets of stators: a cut-on, 22-vane set and a cutoff, 40-vane set. Extrapolations from previously measured data at takeoff conditions indicated that the tone noise from the ADP might be lower than the background noise level in the 8- by 6- Foot Wind Tunnel. Therefore, the noisier, cut-on, 22-vane set was chosen for this 
experiment to improve the likelihood of obtaining cruise noise data. The ADP model was tested at its design cruise blade setting angle with three inlet lengths (Fig. 2). The long and medium length inlets were tested with the short spinner, and the short inlet was tested with the plug spinner. The long inlet has a length-todiameter ratio similar to current turbofan inlets. Figure 1 shows a photograph of the short inlet and plug spinner. Table II is a listing of the configurations tested.

\section{Acoustic Measurements}

Noise measurements were obtained using pressure transducers installed flush with the surface of a plate suspended from the tunnel ceiling. The plate is used instead of the tunnel wall to allow measurements to be made closer to the model and to reduce the boundary layer background noise. The plate surface was $49.8 \mathrm{~cm}$ (19.6 in) above the tunnel centerline, with the tunnel and model centerlines coinciding. The plate was installed in two positions, forward and aft. In the forward position, the plate was roughly centered on the lip of the long inlet, and in the aft position, the plate was centered on the nozzle exhaust plane. Figure 3 is a photograph that was taken during testing with the short inlet and shows the plate installed in the aft position. Figure 4 depicts the acoustic plate locations for the forward and aft positions. The plate contained 12 transducers, the locations of which are listed in Fig. 4. The angular positions of these transducers, measured from various reference planes, are listed in Table III.

The signals from the pressure transducers were recorded on magnetic tape and analyzed after the tests were completed. Narrowband frequency domain spectra were obtained with a range of 0 to $10000 \mathrm{~Hz}$ with a bandwidth of $32 \mathrm{~Hz}$. Zoom spectra within a $312-\mathrm{Hz}$ span and with a resolution of $1.56 \mathrm{~Hz}$ were obtained at some of the test conditions to extract the blade passing tone from the tunnel background. Obtaining enhanced spectra, which were time domain averaged using the fan's once per revolution signal, was also attempted. This effort was not successful because of significant acoustic energy loss, apparently caused by the test rig motion in relation to the fixed acoustic plate. This motion caused varying phase of the tone signal and resulted in the energy loss.

\section{Results and Discussion}

\section{Noise at Cruise Mach Number and Design Speed}

Noise data were obtained at the $M=0.8$ cruise condition at 100 percent design speed and analyzed on both a 0 - to $10-\mathrm{kHz}$ and zoom spectral basis. A typical 0 - to $10-\mathrm{kHz}$ spectrum (Fig. 5(a)) shows the wind tunnel compressor tones and the relationship of the ADP blade passing tone to the underlying tunnel broadband noise level. Various sources of this tunnel broadband noise level include, among others, the tunnel compressor and the wall boundary layer. The tunnel broadband level near the ADP blade passing tone, about $130 \mathrm{~dB}$, limited the observable ADP tone to only the peak levels at a few aft angles. A typical zoom spectra is shown in Fig. 5(b); with a frequency resolution of $1.56 \mathrm{~Hz}$. Spectra with finer resolutions were also computed, but some tone energy was lost, probably because of small rpm variations of the model. The finer resolution of the zoom spectra lowered the broadband level near the tone to about $110 \mathrm{~dB}$ (Fig. 5(b)). This 110-dB level imposes the practical limit for the lowest ADP tone that could be resolved accurately. The criterion often used is that the tone level be $6 \mathrm{~dB}$ or more above the background noise so that its level is accurate within $1 \mathrm{~dB}$.

Noise directivities at $M=0.8$ and 100 percent design speed are shown in Fig. 6. As seen, particularly for the short inlet (Fig. 6(a)), the directivity patterns have peaks corresponding to forward (inlet) and aft (nozzle) radiation sources. The minima between the forward and aft peaks may be exaggerated because of high velocities between the model and the plate. At $M=0.83$ for the short inlet, a condensation shock pattern was seen between the back part of the nacelle and the plate when the plate was in the aft position. The high velocities between the plate and the nacelle keep the noise radiated from the aft duct from propagating forward to the inlet arc. This would separate the forward and aft noise and exaggerate the minima.

The aft noise peak is dominant. Only in this region is the blade passing tone visible on the $0-$ to $10-\mathrm{kHz}$ spectrum. The breaks in the curve on Fig. 6 indicate regions where the blade passing tone was not visible above the tunnel background noise on the zoom spectra; that is, the tone was below $110 \mathrm{~dB}$. The noise in the aft quadrant is about the same in both level and shape for all three inlet lengths, as might be expected. The configuration with the medium inlet had a slightly higher peak noise in the aft and represents the noisiest configuration at this condition. Significant differences in the forward arc noise were observed and will be discussed in the section on variation of inlet noise.

The Advanced Ducted Propulsor has significant advantages over an unducted propeller configuration in reducing the likelihood of cabin and enroute noise problems. Figure 7 shows the noise directivity of the SR-7A propeller compared with the ADP. The noise level of the SR-7A propeller was adjusted by 10 times the log of the net thrust ratio between the SR-7A and the ADP to allow for direct comparison. This adjustment raised the SR-7A levels by $2 \mathrm{~dB}$. The distance from the SR-7A 
propeller and from the ADP model to the acoustic plate were the same.

From Fig. 7, the ADP peak blade passing tone noise is approximately $30 \mathrm{~dB}$ less than the SR-7A peak blade passing noise. Reference 3 estimated the enroute noise of an airplane at $30000 \mathrm{ft}$ with $\mathrm{SR}-7 \mathrm{~A}$ propellers as having a peak of $65 \mathrm{dBA}$. With the ADP peak at $30 \mathrm{~dB}$ lower than the SR-7A peak, the ADP should not represent an enroute noise problem. Because acceptable cabin noise levels of high-speed propellers were viewed as achievable, ${ }^{6}$ the $30-\mathrm{dB}$, lower level of the ADP would indicate the absence of a cabin noise problem. Furthermore, the configuration tested had a cut-on blade passing tone. The candidate production configuration has a cutoff blade passing tone and would be even quieter.

Noise Variation with Fan Speed and Tunnel Mach Number

Figure 8 shows the maximum blade passing tone at $M=0.8$ as a function of fan speed. The noise first increases as the fan speed is increased and then goes down at the 100 percent design speed condition. (The fan thrust showed an almost linear increase with increasing percent speed over the tested range from 85 to 100 percent design speed.) The maximum blade passing tone after the bendover is reduced to almost the level at 85 percent design speed. This bendover has been noted previously for high-speed propellers ${ }^{2}$ when the noise was shown to correlate with helical tip Mach number (the vector sum of axial and rotational Mach numbers at the tip). Figure 9 shows an example of this behavior for the SR-7A propeller. Here, after bending over, the noise has a local minimum before starting to rise again.

Figure 10 shows the ADP data for the medium inlet as a function of helical tip Mach number. Data are shown here for the medium inlet only, but data for the other inlets demonstrate the same behavior. Figure 10(a) shows data at a constant tunnel Mach number of 0.8 with varying fan speed. Figure 10(b) shows the data with varying tunnel Mach number at a fixed 100 percent design speed. Because of the fan duct, the variation in tunnel Mach number from 0.6 to 0.85 results in only small changes in the fan face axial Mach number. This then results in the small range of helical tip Mach numbers plotted on Fig. 10(b). The ADP data show the same general behavior of bending over as did the turboprop data, but the ADP data bend over at a much lower helical tip Mach number. Note here that the SR-7 noise is from a rotor alone source. For the ADP, this source is cut off in the duct since the fan tip speed is subsonic. The rotor-stator interaction is the primary source for the ADP tone noise. This difference in noise source is the probable reason for the different helical tip Mach number of the bendover.
After the noise bendover, the SR-7A levels rose again with increasing helical tip Mach number (Fig. 9), which left a local minimum. The presence of a minimum and the subsequent increase were also seen for the ADP at takeoff conditions (see Fig. 13 of Ref. 5). At the cruise condition presented here, the ADP data were not taken to a high enough fan speed to determine if such a minimum would exist in this situation.

\section{Variation of Inlet Noise with Length of Inlet}

The directivities of inlet noise for 100 percent speed operation of the ADP at $M=0.8$ and $M=0.6$ are plotted in Fig. 11. Values of the blade passing frequency sound pressure level are shown for the three inlets tested. At both Mach numbers, the short inlet had the highest blade passing tone levels for this cut-on fan configuration. At $M=0.8$, the medium and long inlets had approximately the same peak levels, at a value lower than the short inlet levels, and the two inlets had similar directivities. At $M=0.6$, the medium inlet had approximately the same peak level as the long inlet. The medium inlet peak noise was near 40 degrees while the long inlet peak was near 60 . From Fig. 10 of Ref. 5, the data at $M=0.2$ also showed the same trend of the short inlet as the noisiest. The medium inlet for the ADP at $\mathrm{M}=0.2$ was only slightly quieter. Because these lowspeed data (from Ref. 5) were taken with the cut-off version of the ADP at a different blade setting angle, direct comparison with the present data is not possible.

The cruise noise data show the short inlet configuration to be significantly noisier in the inlet arc. The inlet noise for the ADP is, however, far below the peak noise that is located in the aft. Therefore, for this device, the choice of inlet does not significantly affect the peak observed noise. (This may not always be the case for all fans.) These data show a preference for using the long or medium inlet over the short inlet when inlet arc noise is a significant contributor to the total noise.

\section{Summary of Results}

A model Advanced Ducted Propulsor (ADP) was tested in NASA Lewis Research Center's 8- by 6-Foot Wind Tunnel to obtain acoustic data at cruise conditions. The ADP model was designed and manufactured by Pratt \& Whitney Division of United Technologies. The model was tested with three inlet lengths. The fan has 16 rotor blades and 22 stator vanes, which results in a cut-on condition with respect to the fundamental rotor-stator interaction tone. Acoustic data were obtained with pressure transducers mounted flush in a plate suspended from the tunnel ceiling above the ADP model. 
Comparisons of the noise directivity of the ADP with that of a previously tested high-speed, unducted propeller showed the ADP peak blade passing tone was about $30 \mathrm{~dB}$ below that of the propeller with equivalent thrust. This significantly lower noise level indicates that the ADP should not present an enroute or cabin noise problem. Furthermore, the configuration tested had a cut-on blade passing tone, and the configuration with a cutoff blade passing tone would be even quieter.

The maximum blade passing tone first increased with increasing helical tip Mach number and then decreased at higher Mach numbers. This behavior was observed for both variations of fan speed at constant tunnel axial Mach number and variations of tunnel axial Mach number at constant fan speed. Bendover has been observed previously for high-speed propellers but occurred here at a lower helical tip Mach number than that indicated in the propeller data. The ADP tests with the shortest inlet showed more noise in the inlet arc than did tests with either of the other two inlet lengths.

\section{References}

1. Hager, R.D., and Vrabel, D., "Advanced Turboprop Project," NASA SP-495, 1988.
2. Dittmar, J.H., and Stang, D.B., "Cruise Noise of the 2/9th Scale Model of the Large-Scale Advanced Propfan (LAP) Propeller," NASA TM-100175, 1987.

3. Dittmar, J.H., "An Estimate of the Enroute Noise of an Advanced Turboprop Airplane," NASA TM-87302, 1986.

4. Dittmar, J.H., "An Estimate of the Noise Shielding on the Fuselage Resulting From Installing a Short Duct Around an Advanced Propeller," NASA TM-100262, 1988.

5. Woodward, R.P., et.al., "Far-Field Noise and Internal Modes From a Ducted Propeller at Simulated Aircraft Takeoff Conditions," AIAA Paper 92-0371, 1992.

6. Revell, J.D., Balena, F.J., and Koval, L.R., "Interior Noise Control by Fuselage Design for High-Speed Propeller-Driven Aircraft," J. Aircraft, Vol. 19, No. 1, 1982, pp. 39-45.

TABLE I.-ADP DESIGN PARAMETERS

\begin{tabular}{|c|c|}
\hline otor blades & $\cdots \cdots$ \\
\hline $\begin{array}{l}\text { ator vanes } \ldots \ldots \\
\text { age pressure ratio }\end{array}$ & 022 \\
\hline tage pressure ratio $\ldots \ldots \ldots$ & 1.243 \\
\hline $\begin{array}{l}\text { Stage mass flow, } \mathrm{kg} / \mathrm{s},(\mathrm{lbm} / \mathrm{s}) \\
\text { Rotor diameter, } \mathrm{cm} \text { (in.) } \ldots\end{array}$ & $119.9(54.5)$ \\
\hline $\begin{array}{l}\text { Rotor diameter, } \mathrm{cm} \text { (in.) } \ldots \\
\text { Rotor tip speed, } \mathrm{m} / \mathrm{s}(\mathrm{ft} / \mathrm{s})\end{array}$ & $43.81(17.25)$ \\
\hline $\begin{array}{l}\text { Rotor tip speed, } \mathrm{m} / \mathrm{s} \text { (ft/s) } \\
\text { Rotor mid-span chord, } \mathrm{cm} \text { (in.) }\end{array}$ & .. $257(844)$ \\
\hline Rotor mid-span chord, cm (in.) & $7.65(3.01)$ \\
\hline 2-vane stator mid-span chord, & $6.76(2.66)$ \\
\hline
\end{tabular}

22-vane stator mid-span chord, cm (in.) ... $6.76(2.66)$

TABLE II.-CONFIGURATIONS TESTED

\begin{tabular}{|c|l|l|l|}
\hline Configurations & Inlet & Spinner & $\begin{array}{c}\text { Acoustic } \\
\text { plate position }\end{array}$ \\
\hline A & Long & Short & Forward \\
B & Medium & Short & Forward \\
C & Short & Plug & Forward \\
D & Short & Plug & Aft \\
E & Medium & Short & Aft \\
F & Long & Short & Aft \\
\hline
\end{tabular}


TABLE III.-TRANSDUCER ANGULAR LOCATIONS

(a) Angle with plate forward, $\theta$, deg

\begin{tabular}{|r|r|r|r|r|}
\hline \multirow{2}{*}{ Transducer } & \multicolumn{3}{|c|}{ Inlet plane } & $\begin{array}{c}\text { Fan } \\
\text { stacking } \\
\text { axis }\end{array}$ \\
\cline { 2 - 5 } & Long & Medium & Short & \\
1 & 46.5 & 43.6 & 39.2 & 34.2 \\
2 & 49.8 & 46.5 & 41.6 & 36.1 \\
3 & 58.2 & 54.1 & 48.0 & 41.1 \\
4 & 71.7 & 66.6 & 58.6 & 49.5 \\
5 & 79.4 & 73.9 & 64.9 & 54.6 \\
6 & 90.4 & 84.5 & 74.7 & 62.6 \\
7 & 99.6 & 93.9 & 83.7 & 70.5 \\
8 & 103.6 & 97.9 & 87.7 & 74.2 \\
9 & 109.5 & 104.1 & 94.2 & 80.3 \\
10 & 116.4 & 111.5 & 102.2 & 88.4 \\
11 & 119.6 & 115.0 & 106.0 & 92.5 \\
12 & 130.1 & 126.5 & 119.4 & 107.7 \\
\hline
\end{tabular}

(b) Angle with plate aft, $\theta$, deg

\begin{tabular}{|r|c|c|}
\hline Transducer & Nozzle exit plane & $\begin{array}{c}\text { Fan } \\
\text { stacking } \\
\text { axis }\end{array}$ \\
\hline 1 & 46.6 & 78.1 \\
2 & 49.9 & 83.8 \\
3 & 58.3 & 96.6 \\
4 & 71.9 & 112.2 \\
5 & 79.6 & 118.8 \\
6 & 90.6 & 126.7 \\
7 & 99.8 & 132.2 \\
8 & 103.7 & 134.4 \\
9 & 109.6 & 137.5 \\
10 & 116.5 & 141.0 \\
11 & 119.7 & 142.5 \\
12 & 130.2 & 147.7 \\
\hline
\end{tabular}




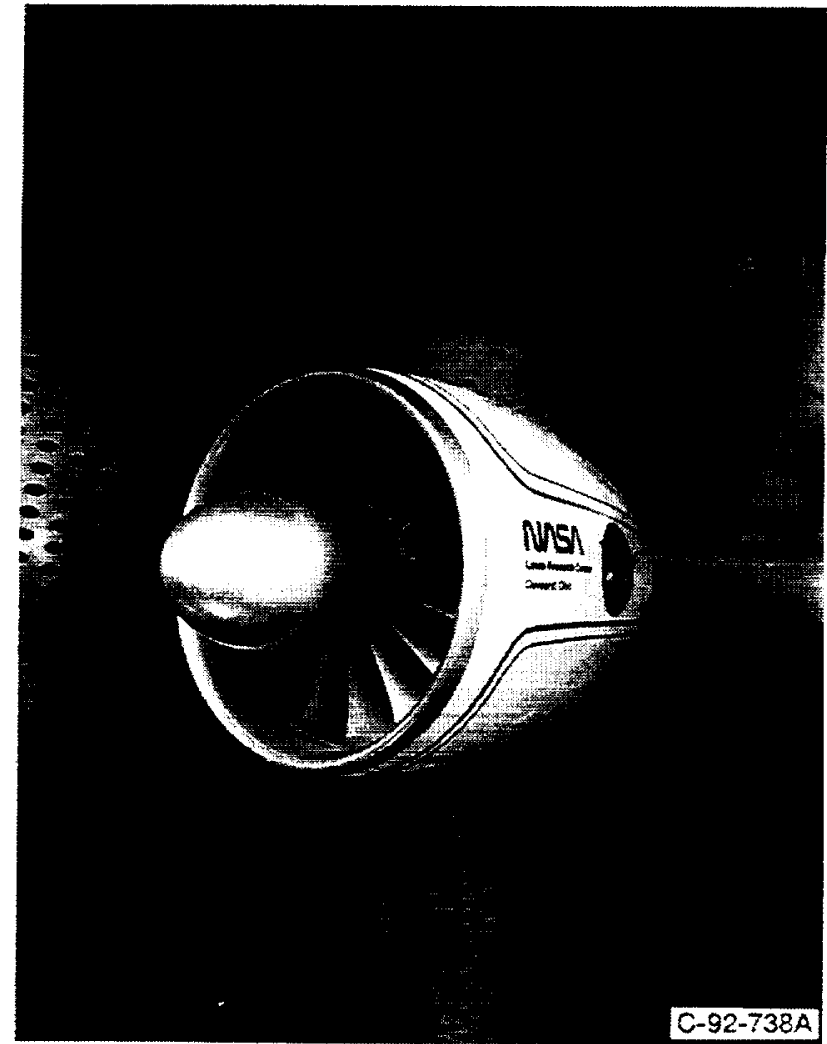

Figure 1.-Advanced Ducted Propulsor (ADP) model.

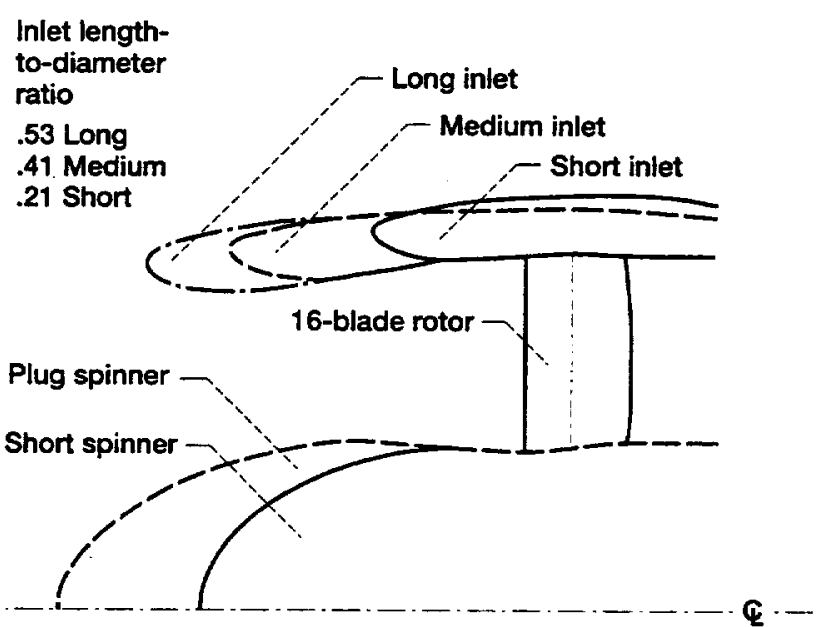

Figure 2.-ADP inlet configurations.

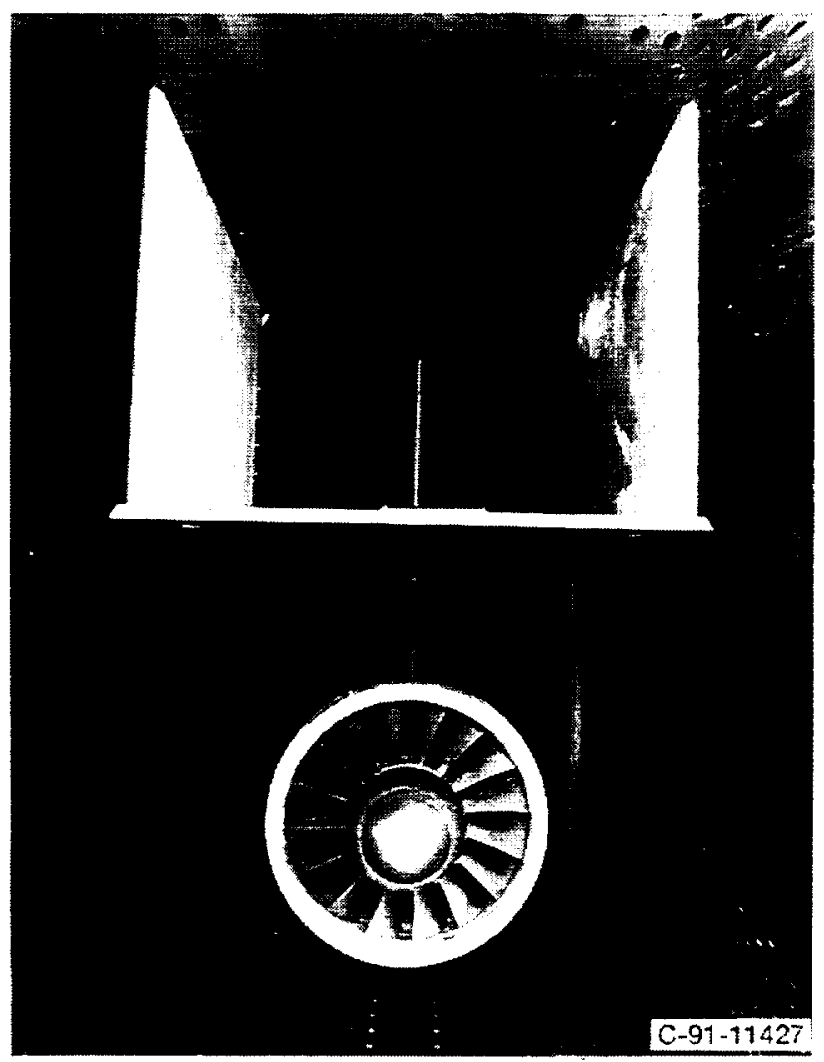

Figure 3.-Acoustic plate in aft position. 


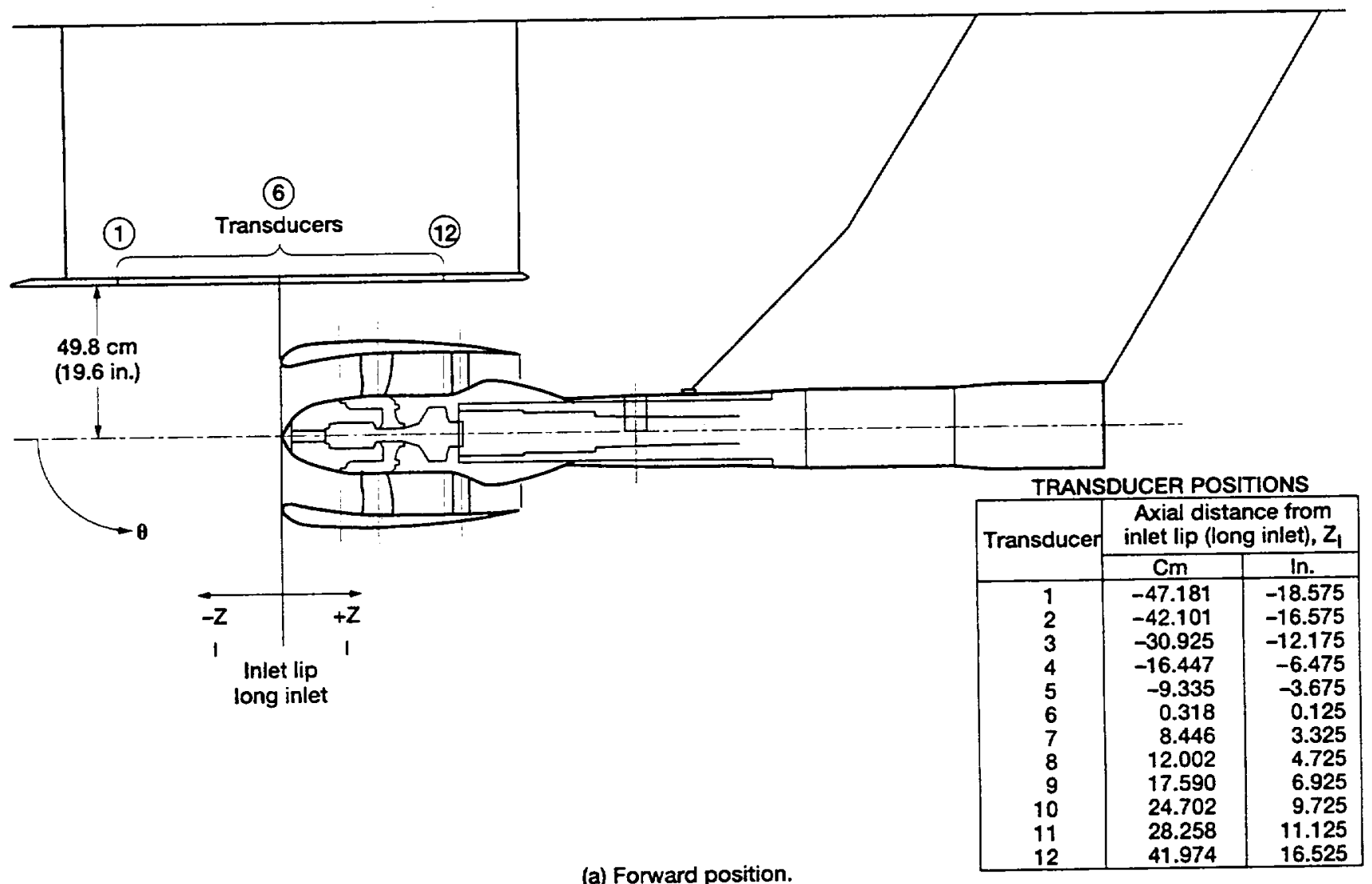

(a) Forward position.

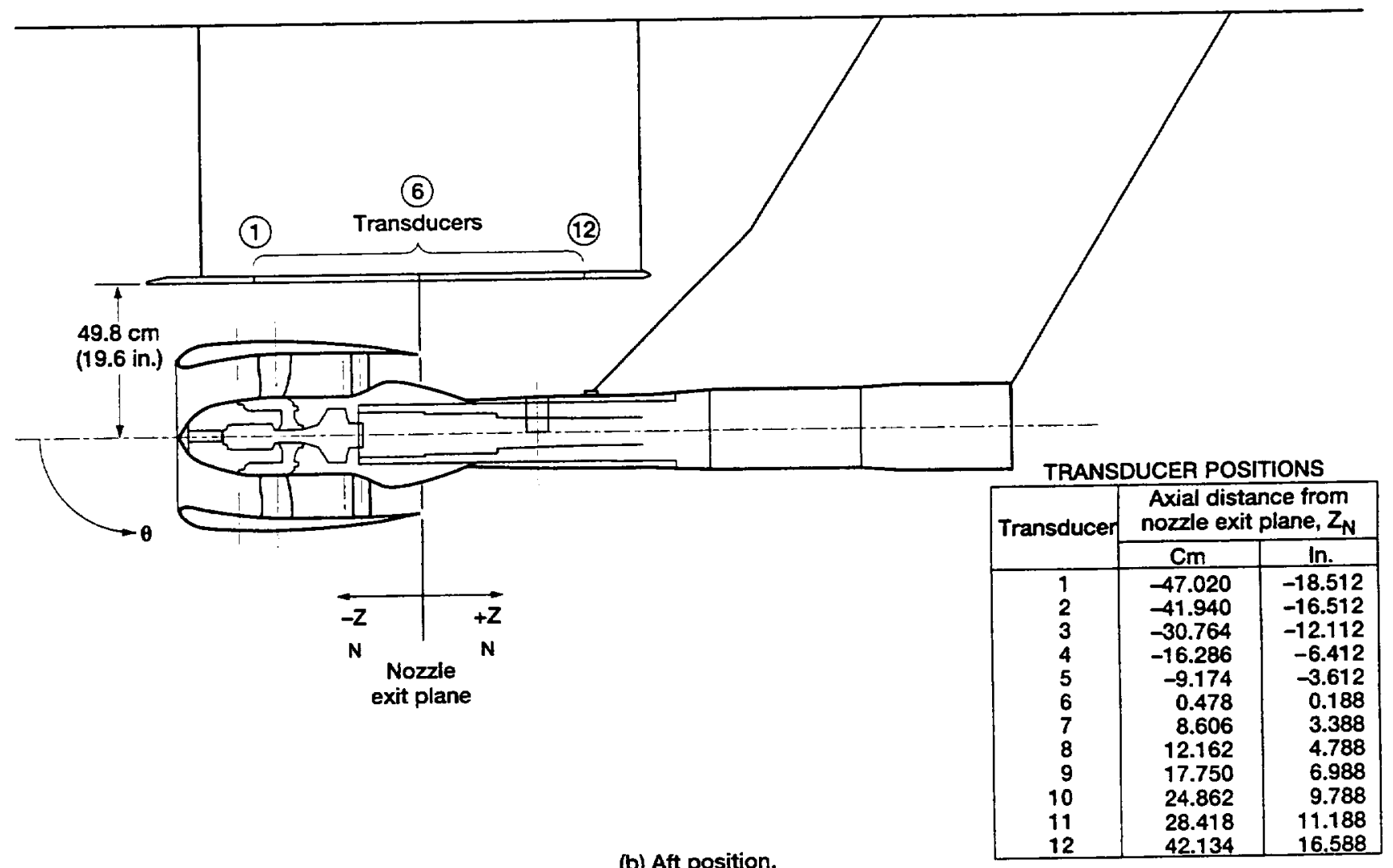

Figure 4.-Acoustic plate positions and transducer locations. 


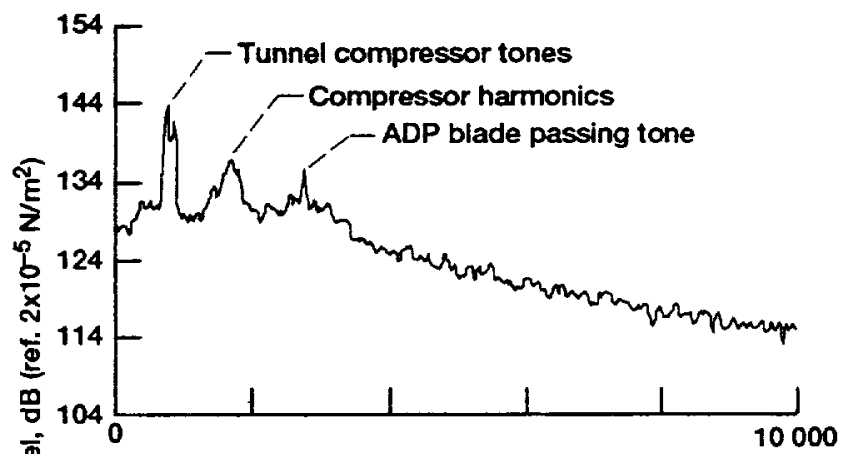

(a) Typical 0 to $10 \mathrm{kHz}$ spectrum when ADP blade passing tone is visible.

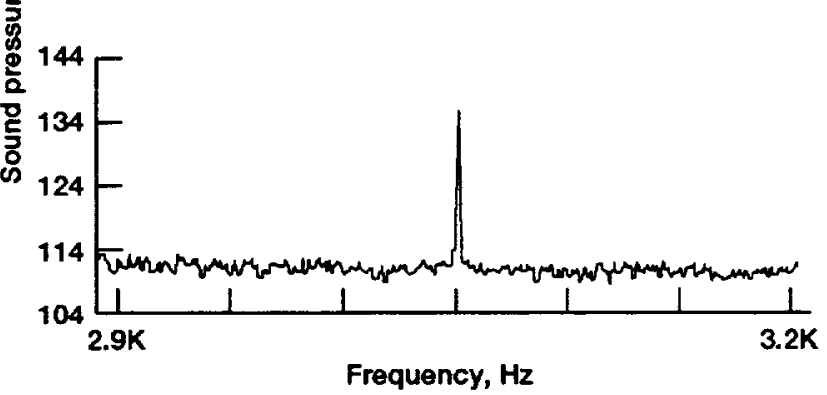

(b) Typical zoom spectra showing ADP blade passing tone.

Figure 5.-Spectra at cruise Mach number and design speed.

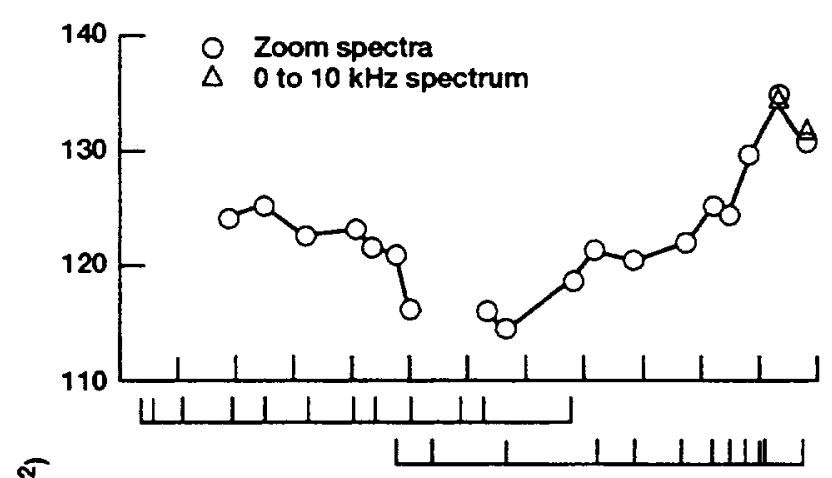

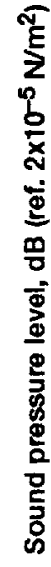

(a) Short inlet.

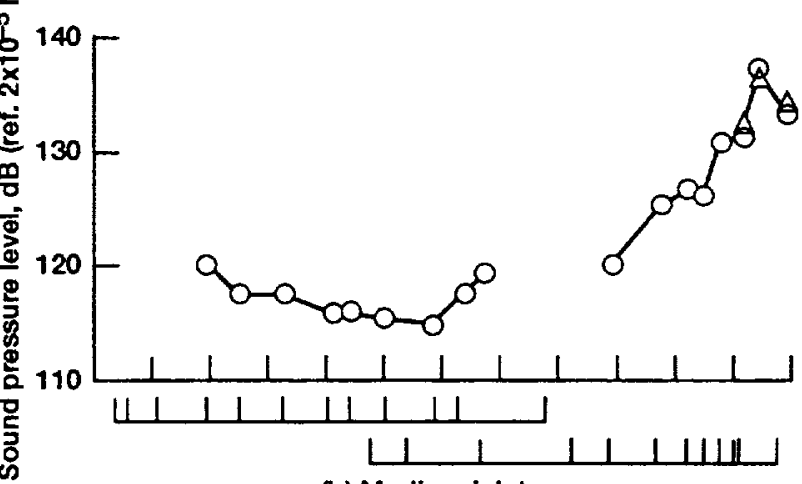

(b) Medium inlet.

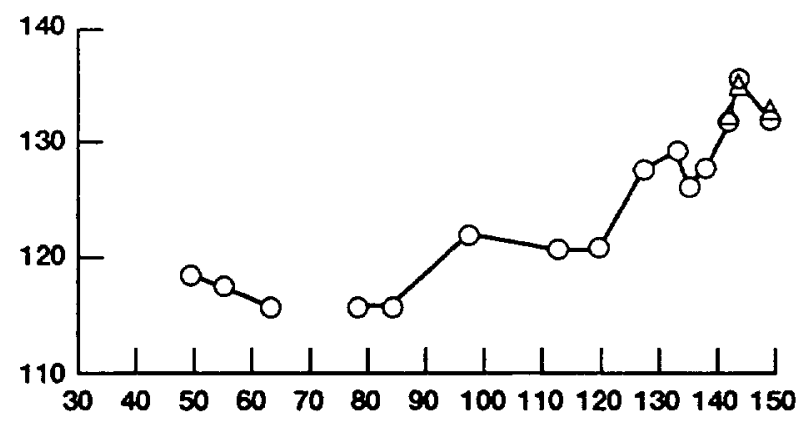

Angle from inlet, centered on fan blade stacking axis

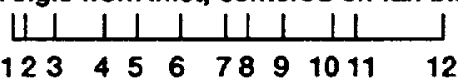

Forward plate transducer positions

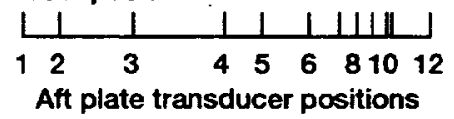

(c) Long inlet.

Figure 6.-Directivities at $M=0.8 ; 100$ percent speed. 


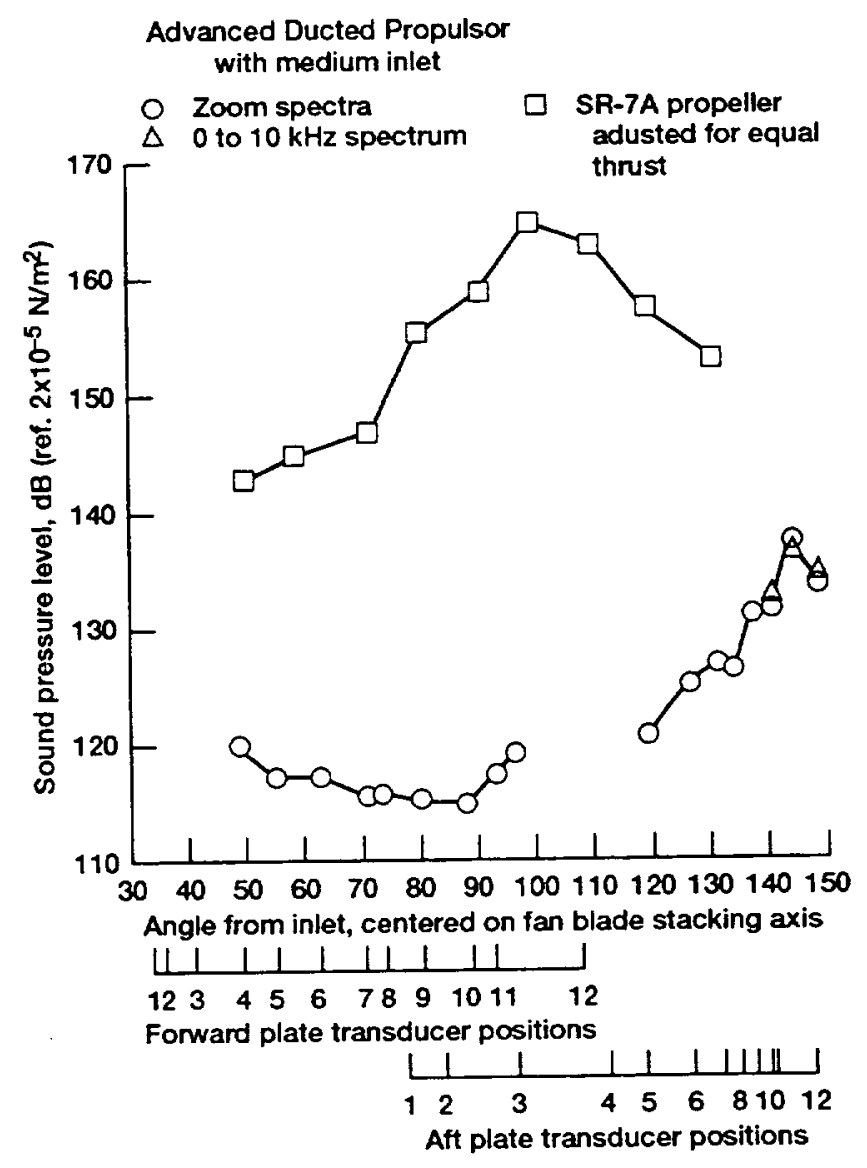

Figure 7.-Comparison of noise levels at $M=0.8 ; 100$ percent speed.

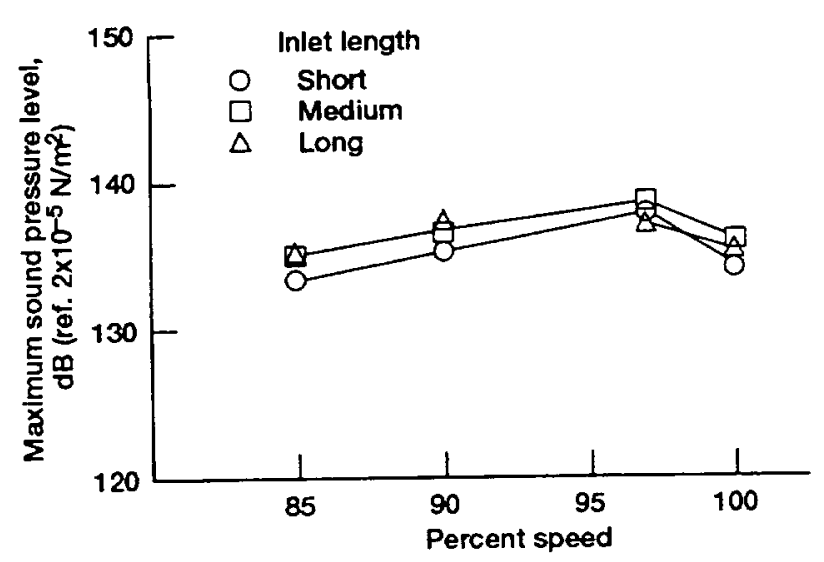

Figure 8.-Variation of maximum blade passing tone with percent speed at $M=0.8$.

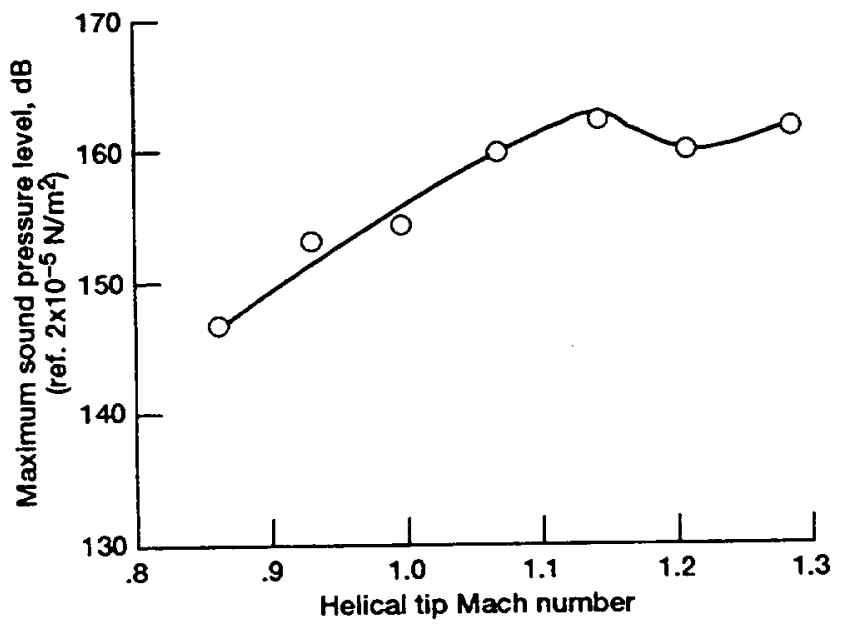

Figure 9.-Maximum blade passing tone variation with helical tip Mach number for the SR-7A propeller at an advance ratio of 3.06 . 


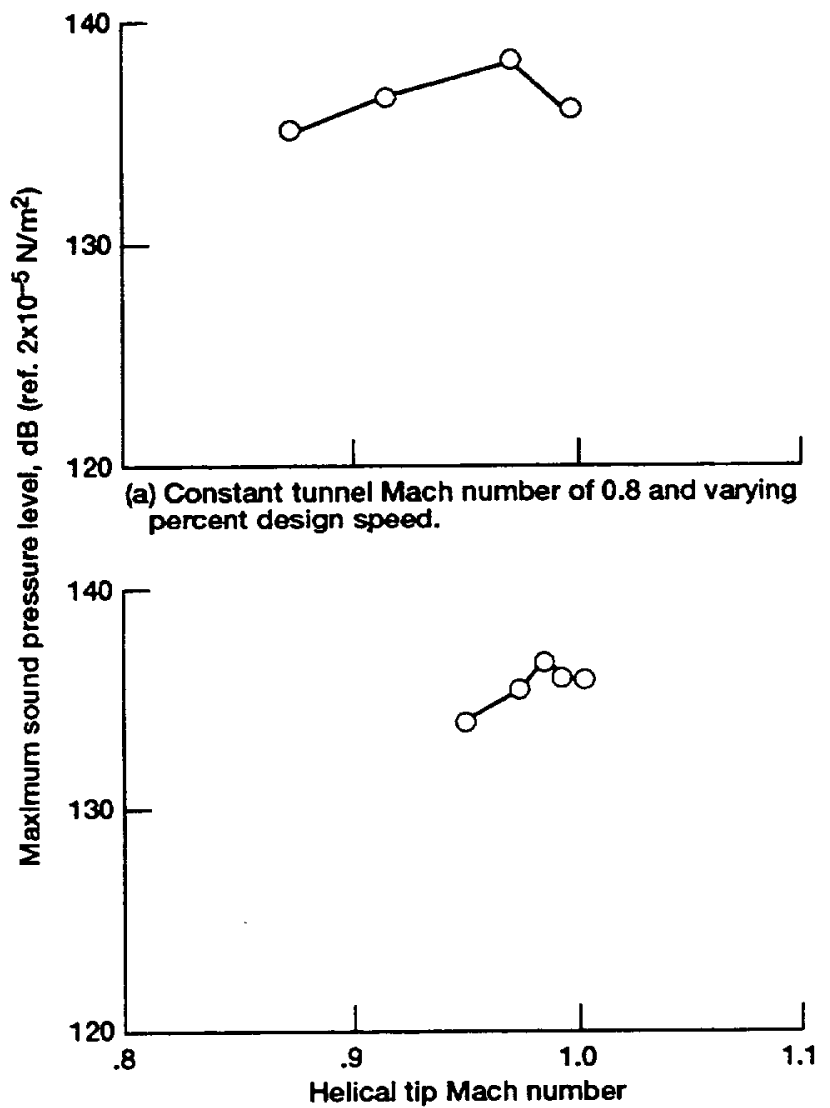

(b) Varying tunnel Mach number and constant 100 percent design speed.

Figure 10.-Maximum blade passing tone variation with helical tip Mach number (medium inlet).

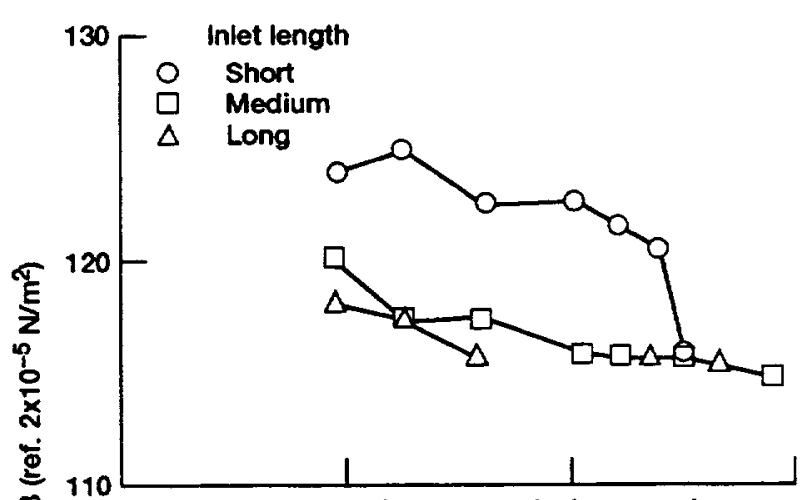

(a) $M=0.6 ; 100$ percent design speed.

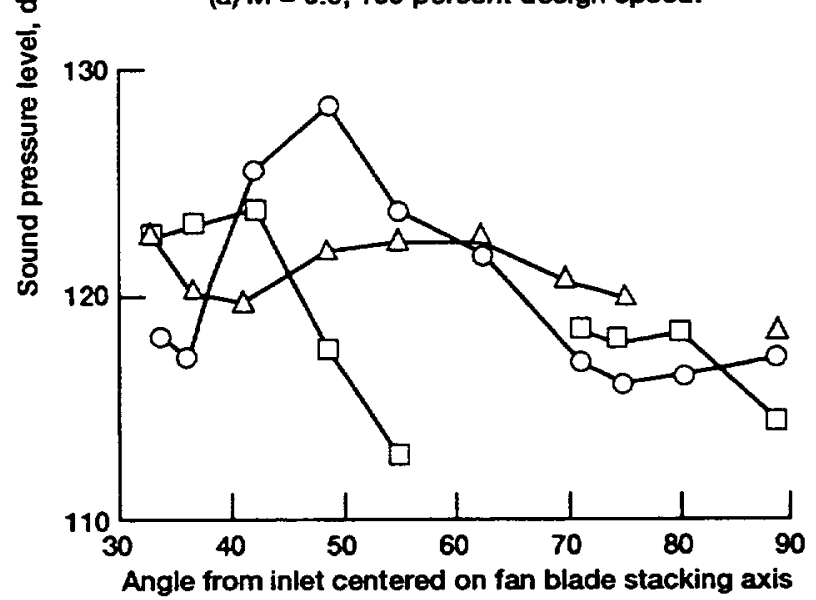
(b) $M=0.6 ; 100$ percent design speed.

Figure 11.-Inlet are blade passing tone with different inlet lengths. 


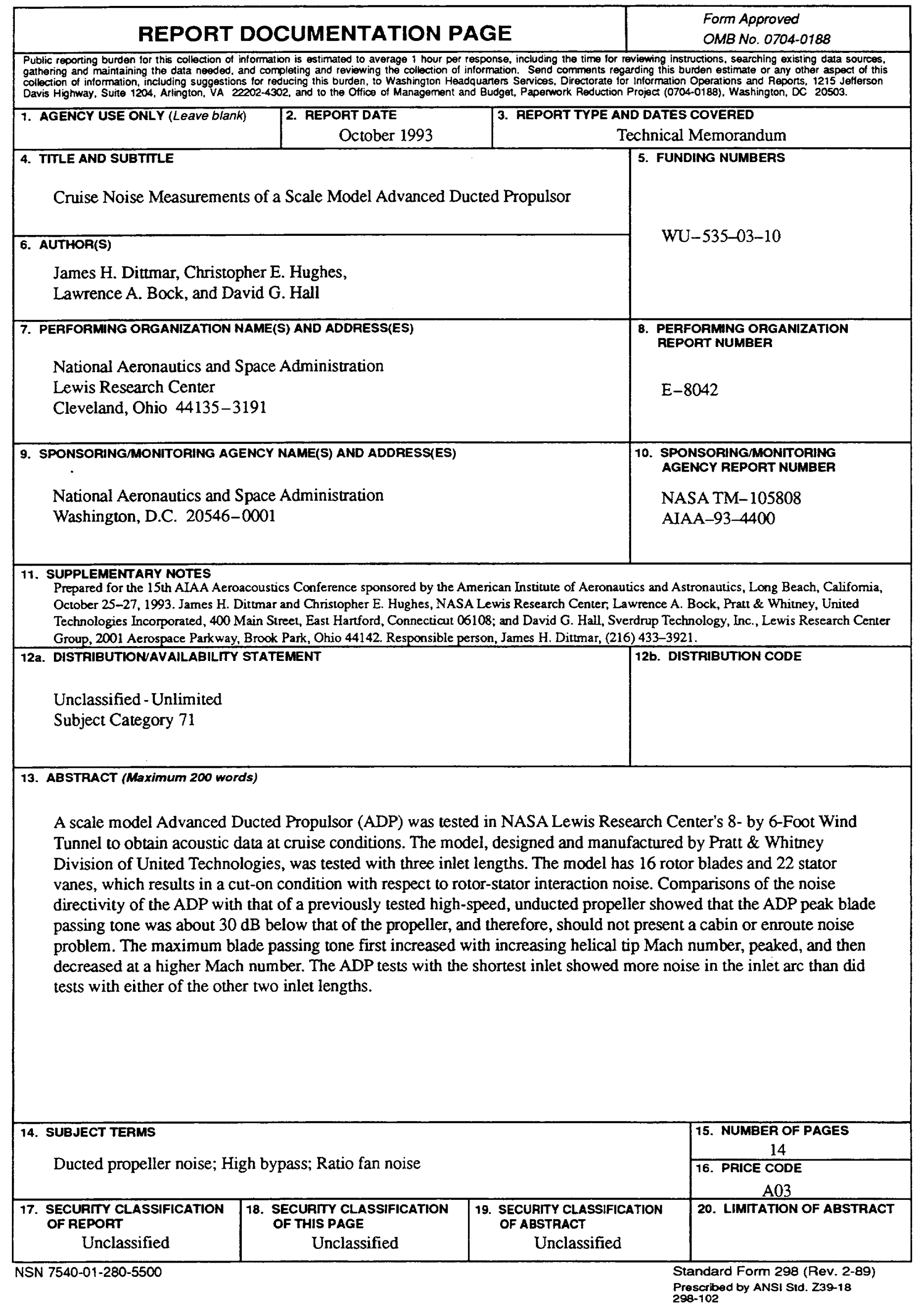

